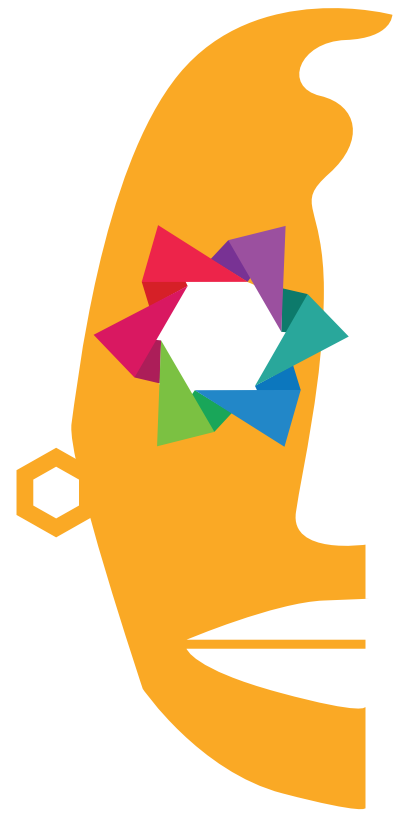

Martín López Ávalos

El Colegio de

Michoacán, A.C.,

Centro de Estudios

Históricos

México

\title{
Élites y poder en Cuba, de la épica revolucionaria a la instrumentalización del Estado, 1959-1965
}

\section{Elites and power in Cuba, from the revolutionary epic to the instrumentalization of the State, 1959-1965}

\section{Resumen}

Se parte de la hipótesis que el Estado nacional cubano es el resultado de la acción de una serie de elites que siempre se han considerado revolucionarias. La construcción del Estado cubano abarca tres experiencias que explican el ejercicio del poder político por elites específicas en el siglo XX. Es la capacidad de agencia de estas elites la que explica la historia política del Estado, independientemente de las intervenciones externas de las que puede ser objeto. Lo que definimos como Revolución cubana, es un proceso que puede distinguirse en dos fases, la insurreccional entendida como la toma del poder político, y la construcción de los mecanismos que permiten la reproducción de la nueva elite como elite de poder, es decir, a través del aparato del Estado. En este proceso particular se observa la importancia de un líder carismático como Fidel Castro, quien logra aglutinar a un conjunto de personas en torno de su liderazgo.

Palabras clave: elites políticas, Insurrección, Cuba política y gobierno siglo XX, Castrismo

\section{Abstract}

It is based on the hypothesis that the Cuban national state is the result of the action of a series of elites that have always been considered 
revolutionary. The construction of the Cuban State includes three experiences that explain the exercise of political power by specific elites in the 20th century. It is the capacity of agency of these elites that explains the political history of the State, independently of the external interventions of which it can be object. What we define as Cuban revolution, is a process that can be distinguished in two phases, the insurrection understood as the seizure of political power, and the construction of the mechanisms that allow the reproduction of the new elite as power elite, that is, to through the State apparatus. In this particular process we can see the importance of a charismatic leader like Fidel Castro, who manages to bring together a group of people around his leadership.

Keywords: Political elites, Insurrection, Cuba politics and 20th century government, Castrismo

No hace mucho tiempo los estudios dedicados a la historia contemporánea de Cuba se ocupaban y se preocupaban de los escenarios de la transición una vez que la figura emblemática de Fidel Castro no está presente físicamente. El inevitable proceso biológico, primero con el deterioro de salud a partir de 2006 del comandante Castro y luego con su muerte diez años después, hizo realidad los sueños más delirantes de la Guerra Fría, ver a Cuba en un periodo de transición, primero pos fidelista y luego poscastrista, cuando el general Raúl Castro, hermano y sucesor de Fidel, anuncia su decisión de retirarse de la actividad política pública como presidente de Cuba en 2018. La transición cubana es una de las más largas, en sentido estricto, abarcando poco más de una década (2006-2018) para ver a un presidente cubano que no solamente no provenga de la misma familia, sino que no pertenece a la misma generación de la elite política que gobierna Cuba desde 1959.

La mayoría de las lecciones que nos deja la transición cubana están todavía por verse y analizarse, sin embargo, lo que sí podemos apreciar es la estabilidad de su sistema político que se mantiene sin cambios significativos desde su gestación y consolidación entre 1959 y $1965^{1}$. El presente ensayo tiene como objetivo mostrar una posibilidad de análisis político para explicar este proceso fuera de los parámetros usuales que se utilizaban durante el periodo de la Guerra Fría,

1 La consolidación de la elite revolucionaria castrista ha sido poco estudiada, salvo excepciones como William Leogrande, The political institutialization of mass-elite linkages in revolutionary Cuba (Syracuse: University of Syracuse, 1976) y aunque muy descriptivo y poco analítico Manuel Sánchez Pérez, ¿Quién manda en Cuba? Las estructuras del poder. La élite (Miami: Ediciones Universal, 1989). Ha sido desde el análisis de la estructura misma del Estado cubano donde las elites ocupan un espacio, por ejemplo, Louis A. Pérez, Cuba Between reform and revolution, 2a ed. (New York: Oxford University Press, 1995); Marifeli Pérez-Stable, The Cuban revolution: Origins, Course and Legacy (New York: Oxford University Press, 1988); Jorge I. Domínguez, Cuba order and revolution (Cambridge: Harvard University-Belknap, 1978). Esta razón nos lleva a concluir que el tema al ser escasamente abordado tiene un mínimo impacto en la historiografía de la revolución cubana como tal. Por esa razón, los estudios utilizados en el presente trabajo no son de "actualidad" ni guardan el canon académico de haber sido publicado en un periodo mínimo diez años para ser considerados "vigentes". Para un análisis sobre las características de la producción historiográfica de la Revolución cubana, véase Martín López Ávalos, «Historiografía de la revolución cubana. Entre los paradigmas y los discursos hegemónicos», en El estudio de las luchas revolucionarias en América Latina (1959-1996) Estado de la cuestión. Edición de Verónica Oikión, et. al. México: El Colegio de Michoacán y Universidad de Santiago de Compostela, 2014. 
donde se le negaba cualquier capacidad de agencia a los actores locales, una vez que se admite que dos grandes potencias hacen y deshacen a su antojo incluso en las encrucijadas nacionales. En vez de eso, mostramos lo contrario, la capacidad de agencia de los actores locales para determinar su propio destino, a partir del control que lleguen a tener del aparato del Estado nacional. La forma en cómo se construye este proceso implica diversas fuerzas políticas como reflejo de una estructura social determinada, donde las elites juegan un papel de primer orden, ya que de ellas se desprende la elite del poder como la instancia encargada del ejercicio del control de todo el aparato del Estado que refleja el ejercicio del poder. A partir de admitir que toda estructura, en cualquiera de sus ámbitos requiere de organización, la acción de las elites cobra mayor significado para explicar la historia política en su conjunto ${ }^{2}$.

En ese sentido, el presente estudio parte de la hipótesis que la formación del Estado nacional cubano es producto de la organización de una serie de elites políticas que siempre se han definido como revolucionarias. Este proceso abarca tres repúblicas, la primera da inicio a la existencia formal del Estado en 1902 y es producto de la guerra de independencia y la intervención norteamericana cuyo corolario está en la Enmienda Platt. La primera república, liberal y oligárquica, carga con ese pecado de origen que marca las disputas políticas de las elites nacionales. La segunda república se produce como una ruptura revolucionaria en contra de la primera en 1933; ahí, además, surge el modelo de la vanguardia como forma de organización para el asalto al poder en contra del principal instrumento de dominación política de la elite oligárquica, compuesta por los veteranos del Ejército Libertador que lucharon en la guerra de independencia, el ejército nacional. La revuelta de los sargentos y activistas universitarios demuestra, por primera vez, la validez de la tesis insurreccional de vanguardia al descabezar al ejército de sus oficiales y tomar los cuarteles en nombre de la revolución que transita acorde a los nuevos tiempos que corren: produce un Estado nacionalista que lo hermana

2 La historia contemporánea de Cuba ha sido vista como la historia del triunfo revolucionario encabezado por Fidel Castro en 1959. En ese sentido, los estudios disciplinarios de la historia y las ciencias sociales han enmarcado esta experiencia no solo como el campo donde se definen los temas, sino los enfoques con los que se tratan aquellos: más que debates teóricos, lo que hemos presenciado han sido discusiones ideológicas a partir de campos políticos definidos por la Guerra Fría, que podemos sintetizar como la visión de la teoría del liberalismo versus la visión marxista y, dentro de ella, la del nacionalismo antiimperialista que en varias ocasiones se han confundido. Esta discusión refleja en campos específicos por disciplina las tensiones ocurridas en la teoría política, sociología e historia para explicar el cambio de las sociedades contemporáneas ocurrido en el siglo XX a través de la democracia y el autoritarismo, como las categorías donde se puede acomodar cualquier discusión que pretenda explicar los fenómenos contemporáneos, entre ellos la existencia de las elites políticas y su importancia como categorías teóricas que explican realidades concretas. Con esto, aspiramos a que la presente colaboración contribuya a un debate disciplinario dentro de las ciencias sociales del presente con instrumentos y categorías derivadas de la teoría política, la sociología y la historia en un tema tan debatido que parece que ya no se puede aportar nada a él, y sin embargo, el tema sigue abierto para ser abordado sin condenas morales contra una "anacrónica dictadura" que insiste en sobrevivir en un mundo donde la teoría liberal pretende hacer válida la metáfora del "fin de la historia". 
con las experiencias más conocidas que se están producido en Argentina, Brasil y México en esos momentos. La tercera república se origina como reacción a la anterior, y se gesta a partir de retomar la herencia insurreccional vanguardista cuando se rompe el orden constitucional en marzo de 1952 con el golpe de Estado, que fue encabezado por Fulgencio Batista. Originalmente su objetivo es la restauración del statu quo anterior al golpe, sin embargo, pronto deviene un nuevo proyecto que la Guerra Fría define como socialista, a diferencia de las dos experiencias anteriores que bordean el marco liberal.

La tercera república corresponde a lo que denominamos Revolución cubana; esta, a su vez, es un proceso en dos fases. La primera es la insurreccional que permite el acceso al poder de una vanguardia organizada política y militarmente en un mismo aparato, que tiene la capacidad de llevar al colapso a un régimen político - que curiosamente tiene su origen en un proceso similar de dos décadas atrás (1933) que reforma al sistema político heredado de la generación de la guerra de independencia-, para dar cabida, en una segunda fase, a la experiencia transformadora más radical conocida hasta entonces por la sociedad cubana, cuando los cimientos mismos de la sociedad liberal fueron derrumbados, primero como una consecuencia del programa político de la vanguardia insurgente que toma el poder y luego, como un imperativo geopolítico al que esta misma vanguardia, convertida ya en elite política, apuesta por sobrevivencia. Todo el proceso de conversión de una forma de organización a otra es el que consideramos necesario explicar para comprender el fenómeno revolucionario que permea la historia contemporánea de Cuba y, en otro sentido, de la historia latinoamericana -e incluso africana subsahariana- de la segunda mitad del siglo XX, por la lectura que diversas fuerzas políticas de esas regiones dieron a los acontecimientos cubanos y los asumieron como lecciones.

La formación de la elite gobernante en Cuba si bien se consolida en el periodo 1959-1965, tiene como antecedente la formación de un movimiento político de vanguardia que deviene en matriz de los miembros de la elite política revolucionaria y posrevolucionaria. En este sentido, veremos cómo existe una relación entre el individuo y el aparato político que, en conjunción con otros elementos, genera el escenario donde la elite política actúa su principal papel: gobernar.

Durante el periodo que va de 1952 a 1959 se conforman las bases para la reproducción de la elite gobernante en $\mathrm{Cuba}^{3}$. En los años previos a la Revolución

3 Podríamos definir a la elite como aquel grupo de personas que ejercen un liderazgo e influencia sobre el resto de la organización política y después, sobre la sociedad. Este problema poco estudiado de la Revolución cubana está planteado en su texto histórico por excelencia, La historia me absolverá. Al anunciar las leyes revolucionarias que se hubieran aplicado de triunfar el asalto al Moncada, Fidel Castro sostuvo que: "La primera ley revolucionaria devolvía al pueblo la soberanía y proclamaba la Constitución de 1940 como la verdadera ley suprema del Estado, en tanto el pueblo no decidiese modificarla o cambiarla, y a los efectos 
encontramos una constante que nos ayuda a entender el ejercicio del poder político después de 1959: alrededor de Fidel Castro como Comandante en Jefe se cierra un círculo de allegados entre colaboradores, seguidores y familiares. La lucha insurreccional, abierta en 1953 con el asalto al cuartel Moncada, se convierte en el filtro de reclutamiento de la verdadera elite que se plasma con la fundación del Movimiento 26 de Julio en 1955, como primera etapa y posteriormente con el Ejército Rebelde en la Sierra Maestra. El Movimiento 26 de Julio legitima primero la formación y el Ejército Rebelde la permanencia en la nueva elite del poder; a partir de estos hechos se conforma una serie de valores que cohesionan a sus miembros. Varios son los rasgos característicos en este proceso; por ejemplo, la fidelidad a la figura del líder, en este caso Fidel Castro y la aceptación de los valores políticos que este implanta como las bases de una nueva "fórmula política", entendida como el proceso a través del cual una elite política justifica su predominio para gobernar al resto de la sociedad. La fórmula política — definida por el sociólogo italiano Gaetano Mosca ${ }^{4}$ como "la base jurídica y moral sobre la que se apoya el poder de la clase política en toda sociedad"- representa un conjunto de valores sociales, políticos y éticos, que permiten a los miembros de una elite diferenciarse de otras al otorgarles unidad y coherencia interna para la acción política, dados los intereses comunes que los une. La fórmula política de la elite emergente tuvo, entonces, como eje articulador la superación de la frustración en lo esencial político: el ejercicio de la soberanía frente a los Estados Unidos ${ }^{5}$.

Paralelamente, la fórmula política castrista insiste en aglutinar a una sociedad fragmentada por las desigualdades sociales generadas por el modelo económico y receloso de las prácticas corruptas de sus políticos. El discurso de restauración democrática encuentra en esos pilares la necesidad de un cambio político que

de su implantación y castigo ejemplar a todos los que la habían traicionado, no existiendo órganos de elección popular para llevarlo a cabo, el movimiento revolucionario, como encarnación momentánea de esa soberanía, única fuente de poder legítimo, asumía todas las facultades que le son inherentes a ella excepto la de modificar la propia Constitución: facultad de legislar, facultad de ejecutar y facultad de juzgar.

"Esta actitud no podía ser más diáfana y despojada de chocherías y charlatanismos estériles: un gobierno aclamado por la masa de combatientes, recibiría todas las atribuciones necesarias para proceder a la implantación efectiva de la voluntad popular y de la verdadera justicia". Véase Fidel Castro, La historia me absolverá. (La Habana: Editora de Ciencias Sociales, 1977), 71. Las cursivas añadidas son para mostrar la idea elitista del líder revolucionario cubano.

4 Gaetano Mosca, La clase política, selección de Norberto Bobbio. (México: Fondo de Cultura Económica, 1984), 132.

5 La referencia con la frustración política está dedicada a la intervención norteamericana, primero en la guerra de independencia que frustra precisamente eso, que las fuerzas por la independencia logren su objetivo, y segundo en la intervención permanente que ese país tuvo, por derecho constitucional vía la Enmienda Platt, en los asuntos internos cubanos hasta su derogación en la presidencia de Franklin D. Roosevelt en 1934. Esta condición de subordinación de toda la clase política cubana al interés de seguridad nacional de Estados Unidos, marca muchas de las características del nacionalismo cubano, en específico su antiimperialismo, como bandera de los grupos políticos que no pertenecen a la elite hasta 1933, cuando sucede la primera transformación al desplazar del poder a la generación de la independencia. 
responda a esos "grandes problemas nacionales". La importancia de una elite política hay que buscarla en esos niveles que reflejan de manera conveniente los valores sociales prevalecientes en esa época y el cómo la elite se los apropia, los representa y se identifica con la masa gobernada ${ }^{6}$. Así, el reclutamiento está abierto para todo aquel que acate estas premisas. El movimiento insurreccional encuentra en la resistencia urbana y posteriormente en la lucha guerrillera, su camino hacia la disputa del poder y su posterior conservación ${ }^{7}$. Los que se integran conforman lo que Robert Michels denomina el "círculo de hierro de la oligarquía" en su ya clásica tesis sobre los partidos políticos. Para Michels toda organización política lleva hacia la oligarquía en su nivel de dirección, es decir, lleva a garantizar la existencia misma de los dirigentes a través de los mecanismos de selección y permanencia en el "aparato".

En el caso cubano, este círculo lo forman hombres y mujeres que desde 1959 aparecen en el primer plano de la política cubana, y que logran permanecer en ese nivel en tres instancias que fueron institucionalizando la carrera política en Cuba.

1. Las Fuerzas Armadas Revolucionarias y el Ministerio del Interior, instancias donde los veteranos de la Sierra Maestra encuentran un lugar para desarrollarse dentro del aparato del Estado.

2. Dentro de la estructura administrativa del Estado en el Consejo de Ministros y, particularmente, su Comité Ejecutivo.

\section{El Buró político del Comité Central del Partido Comunista.}

A continuación, veremos la forma cómo se configura la organización política para construir al sistema político a partir de estas tres instancias que, de una u otra manera, representan el ejercicio del poder político en Cuba. Partimos del hecho que el triunfo de la Revolución, en términos de elites, es un desplazamiento de un nuevo núcleo dirigente que, para permanecer como tal, requiere transitar por

6 Haciendo un recuento de esos años, Fidel Castro admitiría que "Aquella masa no sabía, pero sufría; aquella masa estaba confundida, pero también desesperada. Era capaz de luchar, de moverse en una dirección. Aquella masa había que llevarla al camino de la revolución por etapas, paso a paso, hasta alcanzar la plena conciencia política y plena confianza en su destino" Frei Betto, Fidel Castro y la religión (México: Siglo XXI, 1986), 70.

7 En una carta escrita desde la cárcel, Fidel Castro aborda este problema como un dilema político: "En primer término yo debo organizar a los hombres del 26 de Julio y unir en un irrompible haz a todos los combatientes, los del exilio, la prisión y la calle, que suman más de ochenta jóvenes envueltos en el mismo girón de historia y sacrificio. La importancia de tal núcleo humano perfectamente disciplinado, constituye un valor incalculable a los efectos de la formación de cuadros de lucha para la organización insurreccional o cívica. Es evidente que un gran movimiento cívico y político tiene que tener la fuerza necesaria para ganar el poder por medios pacíficos o revolucionarios; de lo contrario correrá el riesgo de que se lo arrebaten, como a la Ortodoxia, a sólo dos meses de las elecciones" Carlos Franqui, Diario de la revolución cubana, (Barcelona: Ediciones R. Torres,1976), 107.

8 Robert Michels, Los partidos políticos, 2t (Buenos Aires: Amorrortu Editores, 1991), t.I, 189. 
ese proceso que Robert Michels describe para el partido socialdemócrata alemán a finales del siglo XIX y principios del XX. La construcción de la "organización" -con las múltiples ramificaciones que esto implica- se observa en la forma que adquiere el "aparato" entendido como las instituciones que corresponden a este proceso.

A diferencia de otras experiencias históricas socialistas donde el partido es la instancia a partir de la cual se organiza todo el aparato del Estado, en Cuba, por la dinámica propia de la toma del poder, este proceso adquiere particularidades sui generis, pues el mismo partido político gobernante es el resultado de este proceso de organización del aparato del Estado. El Estado socialista en Cuba se origina en un proceso insurreccional donde no existe la conducción del partido socialista como tal, pero sí de un aparato organizado. En la primera década de la Revolución (1959-1969), se observan las formas que adquiere la organización de esta nueva realidad política, pues la elite insurreccional requiere de la construcción del aparato para garantizar las condiciones mínimas de su reproducción como elite dirigente por medio de instituciones para ejercer el poder político. Así, si bien el Partido Comunista de Cuba es fundado formalmente a mediados de la década de los años ' 60 y, tras dos intentos de organización, aparece como institución gobernante, legalizado por una Constitución, una década después cuando se celebra su Primer Congreso en 1975 y se promulga la constitución socialista en 1976.

Resulta fundamental advertir que la creación de la organización política es el resultado de un reacomodo de fuerzas políticas que confluyeron en la profundización de una revolución nacionalista a otra de carácter socialista. El socialismo, a su vez -e independientemente del contexto geopolítico de la Guerra Fría que lo explica- es presentado como la culminación de una lucha de un siglo por la independencia y libertad del país. En ese sentido, y de acuerdo con la lógica política revolucionaria, habría que llegar hasta las últimas consecuencias para aplicar los postulados del nacionalismo cubano. No es extraño que las medidas de profundización y radicalización en contra del capitalismo, cubano y norteamericano, se hayan justificado como una necesidad histórica para alcanzar la independencia nacional. La radicalización a la izquierda del proceso político cubano, paradójicamente, facilita la consolidación de la nueva elite, pues las fuerzas contrarias abandonaron el país dejando el campo libre para la organización de un nuevo sistema político.

En 1961 había tres fuerzas políticas unidas en ese propósito: el Movimiento 26 de Julio, el Directorio Revolucionario y el Partido Socialista Popular (PSP). El primero era la fuerza mayoritaria y de mayor prestigio revolucionario, era la organización insurreccional que había triunfado sobre un ejército profesional y creado las condiciones para este periodo de transición; el segundo era otra fuerza 
insurreccional contemporánea al 26 de Julio, formada por activistas universitarios de la Universidad de La Habana, y el tercero era el partido de los comunistas cubanos, era el único partido -entendido como organización política- que se había sumado a la insurrección en los últimos meses de lucha insurreccional. Con esas tres fuerzas se construiría el nuevo universo político.

El liderazgo carismático de Fidel Castro matiza todas las acciones políticas del nuevo orden, al mismo tiempo que la visión teórica marxista leninista del PSP hace evidente el difícil trance entre cuerpos totalmente diferentes, aunque los uniera su intención final. Para Fidel Castro y sus partidarios, la insurrección es el medio por el que se toma el poder político; para ello se requiere de una organización acorde con una operación de carácter militar. El guerrillero es un soldado no un militante de partido. Al teorizar este proceso, Che Guevara advierte, para salvar la contradicción, que era más fácil que una organización militar de vanguardia marxista (insurreccional) se convirtiera en el embrión de un partido, que este se transforme en un organismo militar para tomar el poder'.

Sin duda, esta forma de tomar del poder es la que imprime las peculiaridades al proceso de organización política, misma que iba contra los principios marxistas leninistas postulados por el PSP. Las dos fuerzas principales que impulsan la construcción del nuevo orden en Cuba no tardan en chocar por este punto. La historia de la organización del partido gobernante cubano no es otra cosa que el desencuentro entre dos visiones de cómo organizar al aparato que garantice la reproducción de la propia elite dirigente. Situación que termina cuando una de estas fuerzas es excluida o absorbida y subordinada por la parte más fuerte.

El primer intento de organizar el aparato político fueron las Organizaciones Revolucionarias Integradas (ORI), donde se observa la clara hegemonía del Movimiento 26 de Julio sobre las otras fuerzas, al mismo tiempo que representa el límite y el quiebre de la coalición que simbolizaron estas tres fuerzas políticas. En esta lucha por el control del aparato es donde cobra importancia el papel de las Fuerzas Armadas Revolucionarias (FAR), pues esta fue el lugar natural donde llegaron los veteranos de la Sierra Maestra; de hecho, podemos decir que el ejército es la primera institucional nacional formada por la revolución, convirtiéndose en el primer aparato del naciente Estado socialista. Gracias a ello, Fidel Castro dispone de los comunistas del PSP opuestos a su liderazgo ${ }^{10}$. Una vez depurado, el partido entra en una asimilación gradual a la égida fidelista hasta diluirse

9 Ernesto Guevara, Obra revolucionaria (México: Era, 1985), 507-514.

10 Previo a esta lucha, el propio Movimiento 26 de Julio es depurado de los militantes moderados quienes, paradójicamente, fueron los primeros en denunciar la creciente "influencia comunista" en el nuevo gobierno. Después del fracaso de la huelga general de mediados de 1958, propuesta por el movimiento urbano, la dirección política se desplaza a la Sierra Maestra; el juicio a Huber Matos en 1959 marca en definitiva la suerte del ala liberal del Movimiento 26 de Julio. Junto a ellos, también fueron desplazados los miembros del 
totalmente. Resulta interesante este proceso, pues la asimilación de los restos del PSP se puede entender por las decisiones políticas de sus dirigentes históricos como Blas Roca y Carlos Rafael Rodríguez, principalmente, quienes aceptan subordinarse al liderazgo de Fidel Castro y demás comandantes revolucionarios. La lucha contra el sectarismo de Aníbal Escalante, denunciado públicamente por el propio Castro es, en otras palabras, la lucha por el control del aparato político de la revolución.

Las FAR, entonces, se convierte en la primera formadora de cuadros en esta etapa; sus más altos oficiales saltan a la burocracia del Estado como después a la dirección del Partido. De acuerdo al razonamiento enunciado por Che Guevara, la vanguardia armada se convierte en vanguardia política. Es pues este soldado irregular, el guerrillero, el modelo de militante que forma a la nueva elite dirigente encabezada por los hermanos Castro.

Al poco tiempo de la disolución de las Organizaciones Revolucionarias Integradas ${ }^{11}$, se anuncia la formación de un Directorio Nacional para organizar al nuevo partido socialista. La composición de este cuerpo es indicativa de la relación de fuerzas al interior del grupo dirigente. De los 25 miembros, 14 provienen del Movimiento 26 de Julio, diez del PSP y uno del Directorio Revolucionario. A su vez, su Secretariado estaba compuesto por seis miembros, cinco de los cuales son del 26 de Julio y uno del PSP. Esta última proporción se encuentra en las otras instancias del Aparato del Estado.

La fundación del Partido Unificado de la Revolución Socialista (PURS) en el verano de 1962 inicia el proceso de institucionalización del ethos revolucionario, identificado por lo que el propio Fidel Castro denomina el "Trabajador ejemplar" como ideal del militante comprometido con la revolución y su liderazgo. En 1965 se da por terminada la organización del partido al anunciarse la fundación del Partido Comunista de Cuba (PCC). Este hecho marca el camino institucional acorde con la nueva legalidad del país. Aunque formalmente el partido adquiere el papel central en la vida institucional del Estado socialista, el peso de las FAR es todavía decisivo, pues los principales cargos dirigentes están ocupados por miembros en activo o que habían pasado a la vida civil de dicha institución, y en un discreto segundo plano -sin carecer de importancia- los militantes sobrevivientes del PSP encabezados por Carlos Rafael Rodríguez y Blas Roca.

gobierno provisional que representaban a la antigua estructura política nacional como el Partido Ortodoxo. La renuncia el presidente Manuel Urrutia y del primer ministro, José Miró Cardona, cierran este círculo.

11 Las ORI fueron creadas en julio de 1961 y disueltas en marzo de 1962 para dar paso al Partido Unificado de la Revolución Socialista de Cuba (PURSC). 
La composición del Comité Central del PCC en 1965 y sobre todo su Buró Político refleja, a su vez, al verdadero "círculo de hierro" que tarda varias décadas en modificarse. Como característica de los miembros de este, tenemos que empalman más de un puesto en una de las instancias donde se toman las decisiones en la administración del Estado ${ }^{12}$.

De acuerdo a este criterio, tres personas tienen un puesto en las cuatro instancias de decisión: Fidel Castro, Raúl Castro y Carlos Rafael Rodríguez. El primero preside todos estos cuerpos, además de ser el Comandante en Jefe de las fuerzas armadas. El segundo es el primer vicepresidente en todas estas instancias, además de ser ministro de las fuerzas armadas. Rodríguez, por su parte, se encarga adicionalmente de las relaciones internacionales. Debajo de ellos, encontramos a 7 personas que ocupan cargos en tres de esas instancias, excepto el Secretariado del Buró Político; todos ellos son, al mismo tiempo ministros de alguna cartera: Ramiro Valdés, Guillermo García, Blas Roca, Pedro Miret, Arnaldo Milián, Oswaldo Dorticós y José Ramón Machado. Otros ocho pertenece a dos de los cuatro: Juan Almeida, Joel Domenech, Flavio Bravo, Diocles Torralba, Belarmino Castilla, Osmany Cienfuegos, Armando Hart y Sergio del Valle. Los dieciocho, obviamente, pertenecen al Comité Central del Partido.

Cabe señalar que, de ese número, la mitad, es decir, 9, lucharon en la Sierra Maestra, uno fue parte de la dirección urbana del Movimiento 26 de Julio; dos se incorporaron a dicho movimiento, aunque no fueron militantes urbanos o guerrilleros; uno fue de las figuras civiles que formarían el primer gobierno provisional.

La militancia, entendida como el camino que se debe seguir (ethos revolucionario) se refleja en el trabajo del Partido o bien en las Fuerzas Armadas Revolucionarias. La estructura política construida por la revolución refleja una gran estabilidad en la medida en que los miembros de la elite política son el resultado de estas condiciones de acceso y permanencia alrededor del Comandante en Jefe.

Si trazáramos varios círculos alrededor del centro político, tendríamos que en el estrato principal aparecen los "históricos" ${ }^{13}$, los que han estado con Fidel Castro

12 Las instancias de decisión del Partido están en el Buró Político del Comité Central y sobre todo su Secretariado; en la administración del Estado tenemos el Comité Ejecutivo del Consejo de Ministros y el Consejo de Estado de la Asamblea Nacional.

13 Aquí hay que abrir otro sub apartado, el de los amigos de la infancia de Fidel Castro; tenemos el caso de Baudilio Castellanos, amigo personal desde el pueblo natal de ambos, Birán en Oriente, compañeros desde la Primaria en el Colegio de Dolores y La Salle en Santiago, donde ambos conocen y traban amistad con Jorge Papito Serguera. Los tres estudian Derecho en la Universidad de La Habana y se hacen abogados. Bilito, como se conoce a Baudilio, es el abogado defensor de la mayoría de los atacantes del Moncada, incluido Raúl Castro, y al triunfo revolucionario transita por diversos cargos de relieve como embajador en Francia y director de empresas estatales. Papito Serguera se convierte en militante del Movimiento 26 de Julio en Santiago y a lo largo de 1959 aplica a pie juntillas la consigna de "Paredón" contra todos los acusados de ser contrarrevolucionarios en su calidad de Fiscal revolucionario. Al igual que Bilito es también 
desde la etapa conspirativa en los años '50, los del Moncada: Raúl Castro, Pedro Miret, Ramiro Valdés, Juan Almeida, Jesús Montané, Melba Hernández y Haydée Santamaría. A estos se suman los principales dirigentes del Movimiento 26 de Julio y del Ejército Rebelde en la Sierra Maestra, como Celia Sánchez, Armando Hart, Vilma Espín, Faustino Pérez y los comandantes y oficiales serranos, donde inevitablemente tiene que incluirse a Ernesto Che Guevara y Camilo Cienfuegos, pero también al primer campesino reclutado del Ejército Rebelde, Guillermo García, así como a otros que han estado momentáneamente, tal es el caso de los comandantes Efigenio Ameijeiras, Universo Sánchez, William Gálvez y los futuros internacionalistas en América Latina y África, desde Raúl Menéndez Tomassevich hasta Arnaldo Ochoa. Ellos se convierten en el pilar del régimen, logrando extender su influencia en puestos de responsabilidad en forma personal y por las redes que se empiezan a tejer a su alrededor. En este sentido, todos los que pertenecen al círculo de los históricos pasan por un reclutamiento hecho personalmente por Fidel Castro, desde esos años de la conspiración para el frustrado ataque al cuartel Moncada, la fundación del Movimiento 26 de Julio; pero en la etapa de la Sierra Maestra la atribución se amplía a Raúl Castro desde su puesto de comandante en la Sierra Cristal. La virtud de este "círculo" ha sido mantenerse en torno al liderazgo de Fidel Castro - pero también de Raúl-y articular sus capacidades individuales en torno a un objetivo común, siempre trazado por Fidel.

El segundo estrado lo componen los sobrevivientes del PSP a las purgas de los años 1961-1962 cuando una parte de su dirigencia enfrenta la hegemonía de Fidel Castro al frente de la revolución. La parte castrista del partido (Carlos Rafael Rodríguez, Fabio Grobart, Blas Roca, etc.) se mantienen al margen de la disputa, aun cuando Fidel Castro arremeta con todas sus fuerzas contra el viejo Partido Socialista Popular. Por último, hay que añadir a los comandantes del Directorio Revolucionario de Estudiantes, Faure Chomón y Rolando Cubelas, y muchos otros que encuentran cabida en el aparato de seguridad del Estado, sobre todo en el Ministerio del Interior con José Pepe Abrantes y en las propias Fuerzas Armadas Revolucionarias.

En estos años, en la elite revolucionaria predominan los hombres, blancos y de clase media como origen social, aunque sí es necesario mencionar que el comandante Juan Almeida es el único afrocubano en este nivel, al igual que el comandante Guillermo García es el único campesino de la Sierra Maestra. Por parte de

embajador, pero en la estratégica Argelia. Existe otro caso, el del hijo del telegrafista de Birán, de apellido Valero, quien se encuentra viviendo en La Habana en el momento de la organización del ataque al Moncada. Valero es de los primeros reclutados por Fidel Castro dado ese lazo con el pueblo común, pero se convierte en una de las primeras desilusiones políticas al volverse informante de la policía; con sus reportes, la policía cubana confisca el mimeógrafo donde se imprime un boletín de agitación llamado El Acusador, y junto con él apresan Jesús Montané y Abel Santamaría, quienes pasan unos días en la cárcel. Véase Ignacio Ramonet, Fidel Castro. Biografia a dos voces (México: Debate, 2006), 99. 
las mujeres, todas tienen el mismo origen social y racial de los hombres de la elite: blancas de clase media. Este primer círculo es muy importante para entender las redes de reclutamiento que se extienden hacia abajo, ya sea por jerarquía política, burocrática o por cambio generacional a partir de 1959. Destaca en esta tarea Raúl Castro, quien desde los tiempos del exilio en México donde se hizo la preparación del primer núcleo guerrillero que embarca en el yate Granma rumbo a las costas cubanas a finales de 1956, tuvo un papel de promotor; no en vano propuso a Fidel Castro la incorporación de Ernesto Guevara a la expedición; ya en la Sierra Maestra se destaca como un comandante bien organizado en cuya columna los reclutados se forman como guerrilleros y después como cuadros políticos y militares que empiezan a ocupar puestos importantes, desde la formación del gabinete presidencial de Manuel Urrutia en 1959. Al ser nombrado Ministro de las Fuerzas Armadas Revolucionarias en octubre de ese año, su papel como promotor se incrementa pues cuenta con el control del principal productor de cuadros del Estado cubano, esto es las Fuerzas Armadas Revolucionarias. Su papel como el número dos en la jerarquía se consolida en estos años. Los comandantes Ramiro Valdés y Juan Almeida ocupan un lugar relevante en esta estructura; nunca han estado subordinados a Raúl Castro y además forjaron su propio prestigio como revolucionarios en el asalto al Moncada, el exilio mexicano, el desembarco del Granma y luego en la construcción del Ejército Rebelde. Almeida es, junto con Raúl Castro, uno de los tres capitanes de las columnas iniciales previstas en el desembarco a las costas cubanas; ya en la Sierra Maestra, también estuvo al frente de una columna guerrillera como comandante y desde entonces su carrera está asociada al mando de tropas y luego de las estructuras burocráticas del Estado como ministro. Valdés, por su parte, sobrevive al desembarco y ya en la Sierra Maestra forma parte de la columna invasora del Che Guevara y entra como su segundo al mando en la fortaleza militar habanera de La Cabaña en los primeros días de enero de 1959; su carrera se relaciona con las funciones de la seguridad, primero del propio ejército y luego como ministro del Interior.

Un lugar destacado en este nivel lo tiene Celia Sánchez. Como coordinadora del Movimiento 26 de Julio en Manzanillo, le toca estar muy de cerca en la gestación del Ejército Rebelde. En la Sierra Maestra se convierte en la mujer con más influencia política; siempre al lado de Fidel Castro actúa como una jefa de gabinete en la guerra y en la paz. Es ella la que coordina en ese periodo de la Sierra, la agenda de Fidel Castro y la que controla mucha de la información que le llega al líder; en estos años, su opinión siempre es valorada y respetada. Las otras mujeres de la elite, las históricas Haydée Santamaría y Melba Hernández, así como Vilma Espín, desarrollan sus propios espacios de control, donde promueven las carreras de nuevos cuadros, sobre todo de la siguiente generación. Santamaría desde la dirección de la Casa de Las Américas; Espín al presidir la Federación 
de Mujeres Cubanas y casarse con Raúl Castro, mientras que Melba Hernández ocupa junto Jesús Montané, su esposo, una oficina al lado de la de Fidel Castro. Sin cargo formal, ambos cuentan como una fuerte influencia política, tanto sobre otros miembros de la elite como en los niveles subordinados.

Sobre los comandantes que ganaron sus grados en la Sierra Maestra y que no pertenecen al círculo histórico, Camilo Cienfuegos y Ernesto Che Guevara estaban destinados a ocupar espacios importantísimos en la toma de decisiones, como fue hasta el momento que vivieron ya integrados a este selecto grupo. Con su muerte muchos de sus subordinados tuvieron que buscar acomodo entre los otros comandantes y ministros; de haber sobrevivido por más tiempo se hubieran colocado al lado de los históricos en cuanto a su influencia y poder dentro de la elite, sobre todo Cienfuegos quien es uno de los personajes más pintorescos y emblemáticos que representaban al nuevo grupo en el poder; su muerte en octubre de 1959 lo priva de continuar por la senda que ya se construye desde los primeros días de ese año. Sin embargo, las circunstancias que envuelven este acontecimiento no dejan de ser sintomáticas en otro sentido, en el de expresar los disensos entre los comandantes de la Sierra Maestra y dirigentes del Movimiento 26 de Julio en contra del proceso mismo, por los términos en que se está llevando en la concentración del poder en Fidel Castro y la incorporación de los comunistas cubanos del PSP en las tareas estratégicas del gobierno, como la reforma agraria, asunto en el cual el comandante Huber Matos se niega a ejecutarlas en la provincia de Camagüey, donde ejerce como máxima autoridad militar. El conflicto que plantea el comandante Matos al liderazgo de Fidel Castro es el primero que viene del interior del grupo de comandantes veteranos de la Sierra Maestra y supone un reto de la misma magnitud, aunque con diferente grado de complejidad política, al que más adelante le proyecta una parte de sus aliados comunistas del PSP con Aníbal Escalante y el proceso contra el sectarismo en 1962. Esto es, una disputa por el control del proceso y del aparato, aunque en el caso de Matos no pasa de una conjetura o posibilidad que no ocurre en su momento, pues tanto los comandantes del ejército rebelde como la tropa a sus órdenes se mantienen fieles al mando principal que encarna Fidel Castro, al mismo tiempo que el comandante Matos no busca liderazgo alguno en ese sentido. El desacato de Matos de aplicar la reforma agraria, sin embargo, muestra la fuerza del aparato militar frente a las estructuras civiles, como el ente encargado de esta tarea, el Instituto Nacional de la Reforma Agraria, y la dirección local del Movimiento 26 de Julio, que se limi$\tan$ a testimoniar su propia impotencia.

El caso del comandante Guevara, por su parte, también muestra otro tipo de fisura al interior de esta misma elite y otra forma de solución. Al contrario de Huber Matos, que termina cumpliendo una condena de 20 años en la cárcel, el 
comandante Guevara expresa una disidencia de diverso tipo y tono, increpa la alianza con la Unión Soviética después de la crisis de los misiles en 1962 y, pese a ello, mantiene su calidad de miembro de la elite como ministro de industrias de Cuba, por lo menos un par de años más cuando abierta y públicamente hace la crítica a la ayuda soviética en un seminario de solidaridad afroasiática organizado en Argelia en febrero de 1965, justo cuando está por cerrarse la organización del nuevo partido comunista y con él, la arquitectura del sistema político de la revolución cubana. La crítica del comandante Guevara a la Unión Soviética es la culminación de un proceso paulatino de alejamiento de Cuba; sus ausencias se hacen cada vez más evidentes, como en este caso que al amparo de su actividad en el foro de Argel, recorre varios países subsaharianos en un periodo de tiempo que ningún ministro puede hacer sin desatender su encargo oficial. Mientras él recorre África, otros se encargan de negociar las condiciones de un nuevo tratado de ayuda soviética para Cuba, lo cual hace evidente que su poder e influencia en la elite revolucionaria de ese país ha terminado. Al retornar a Cuba después del desaguisado en Argel, se enfrenta a la oposición de los comandantes encabezados por Raúl Castro, quien le recrimina sus dichos y las consecuencias que eso trae para la relación de Cuba con su principal aliado. Aislado en sus posturas al interior de la elite cubana, aguantando la ambigüedad de Fidel Castro que no apoya pero tampoco condena, el comandante Guevara quema sus naves y renuncia a todo vínculo legal con el Estado cubano -"nada legal me ata a Cuba", dice en su famosa carta de despedida dirigida a Fidel Castro- para realizar lo que el dirigente cubano ya no puede hacer14. El comandante Guevara deja Cuba, de manera clandestina, poco antes de que se anuncia la composición del Comité Central del Partido Comunista de Cuba. Con la salida de Guevara se cierra un ciclo que coincide con el inicio de la institucionalización al inaugurar las estructuras que la definen como elite gobernante.

La institucionalización del poder revolucionario se da por etapas hasta culminar con la completa formación del partido político marxista-leninista en 1975. Siguiendo esta tradición teórica, éste es un aparato elitista. Pertenecer a él lleva a la aceptación de un proceso de eliminación que deja fuera a la gran mayoría de la población. A su vez, acceder a la dirección de éste trae otro proceso de selección que es igual de discriminatorio que el primer filtro. En el caso de los partidos comunistas, su organización característica ubica al Comité Central como la instancia donde se ejerce el liderazgo político; a su vez, dentro del comité, existe un buró político donde se concentra la verdadera influencia de la dirigencia. En estricto sentido, es la verdadera elite de poder dada su influencia sobre el resto del Partido y, por ende, el aparato del Estado y la sociedad.

14 Guevara, Obra..., 663. 
Los miembros de estas instancias son los que toman las decisiones más importantes en la dirección del país, huelga decir que se empalman en varios puestos a la vez. Haciendo un recuento muy somero, podríamos decir que en Cuba los hombres y mujeres con "influencia" política no sobrepasan las dos docenas. El seguimiento de estas personas: antecedentes familiares, sociales, culturales y, sobre todo, relaciones políticas, resulta de vital importancia para entender el funcionamiento de esta estructura política dominada por un solo hombre pero que necesita de ellos para gobernar.

\section{Bibliografía}

Betto, Frei. Fidel Castro y la religión. México: Siglo XXI, 1986.

Castro, Fidel. La historia me absolverá, versión revisada. La Habana: Editora de Ciencias Sociales, 1975.

Domínguez, Jorge I. Cuba order and revolution. Cambridge: Harvard University-Belknap, 1978.

Franqui, Carlos. Diario de la revolución cubana. Barcelona: Ediciones R. Torres, 1976.

Guevara, Ernesto. Obra revolucionaria, Selección y prólogo de Roberto Fernández Retamar, 10a. ed. México: Era, 1985.

Michels, Robert. Los partidos políticos, 2t. Buenos Aires: Amorrortu Editores, 1991.

Mosca, Gaetano. La clase política, selección de Norberto Bobbio. México: Fondo de Cultura Económica, 1984.

Leogrande, William. The political institutialization of mass-elite linkages in revolutionary Cuba

Syracuse: University of Syracuse, 1976.

López Ávalos, Martín. «Historiografía de la revolución cubana. Entre los paradigmas y los discursos

hegemónicos», en El estudio de las luchas revolucionarias en América Latina (1959-1996) Estado de la cuestión. Edición de Verónica Oikión, et. al. México: El Colegio de Michoacán y Universidad de Santiago de Compostela, 2014.

Pérez, Louis A. Cuba Between reform and revolution, 2a ed. New York: Oxford University Press, 1995. 
Pérez-Stable, Marifeli. The Cuban revolution: Origins, Course and Legacy. New York: Oxford University Press, 1988.

Ramonet, Ignacio. Fidel Castro. Biografía a dos voces. México: Debate, 2006.

Sánchez Pérez, Manuel. ¿Quién manda en Cuba? Las estructuras del poder. La élite. Miami: Ediciones Universal, 1989.

Zambrano, M. (1980). Filosofia y poesía. México: ed. Fondo de Cultura Económico. 